\title{
Caracterização hidrogeológica na região costeira de Lauro de Freitas utilizando o método da eletrorresistividade
}

\author{
Oliveira, R.B., CPGG-UFBA; Vasconcelos, S.S., CPGG-UFBA; Cerqueira, A.G., CPGG-UFBA; Botelho, M.A.B., CPGG-UFBA
}

Copyright 2018, SBGf - Sociedade Brasileira de Geofísica.

Este texto foi preparado para a apresentação no VIII Simpósio Brasileiro de Geofísica Salinópolis, 18 a 20 de setembro de 2018. Seu conteúdo foi revisado pelo Comite Técnico do VIII SimBGf, mas não necessariamente representa a opinião da SBGf ou de seus associados. É proibida a reprodução total ou parcial deste material para propósitos comerciais sem prévia autorização da SBGf.

\section{Abstract}

The coastal area of Vilas do Atlântico is a very wellpopulated area and the hydrology knowledge is of great important to groundwater well for the local population consumption. Located on Lauro de Freitas City, in the state of Bahia, the study area is next to two important rivers that cut the city, the Joanes and Sapato rivers, shown in this research. We adopt electrical surveys to acquire information from subsurface and delineate the possible aquifer zone. From previous studies we had two SEV's (Vertical electrical soundings). Supplies of stratigraphic data from water wells produced by CERB (Companhia de Engenharia e Recursos Hídricos da Bahia) were applied to correlated interpretation of three ERT's (Electrical Resistivity tomography). Then we could delineate lateral variations inside the fractured aquifer evidenced by banding resistivity contrasts.

\section{Introduction}

A cidade de Lauro de Freitas está localizada no nordeste da Bahia, possui área de aproximadamente $60 \mathrm{~km}^{2}$ e faz divisa com três municípios. Ao sul com Salvador, pela praia de Ipitanga e o Aeroporto Internacional de Salvador. $O$ rio Joanes que deságua na praia de Buraquinho delimita a divisa ao norte, com Camaçari. A oeste está a cidade de Simões filho, a estrada CIA-Aeroporto estabelece a divisa entre os municípios. A leste está o Oceano Atlântico, onde situam-se as praia de Ipitanga, Buraquinho e Vilas do Atlântico, local onde foi feito o estudo geofísico. A bacia do Joanes-Ipitanga é parte do sistema de abastecimento humano de água potável da Região Metropolitana de Salvador

A água é indispensável para a existência da vida, o uso racional deste recurso é fundamental para que o suprimento da geração atual e futura seja garantido. $\mathrm{Na}$ região Nordeste do Brasil, sua escassez é mais evidente devido aos longos períodos de estiagem em algumas cidades do sertão, sendo necessário a utilização de meio alternativos como caminhões "pipa" para o abastecimento das cidades. Apesar do drama hídrico ser latente nesta região, foi a recente veiculação midiática da crise hídrica em São Paulo, no sudeste do país, que chamou à atenção de autoridades e governos para a necessidade do mapeamento dos aquíferos subterrâneos em regiões que sofrem com a seca. Conhecer melhor os recursos da subsuperfície torna possível traçar metas e planos para seu uso e preservação. A geologia da região está relacionada à bacia sedimentar do recôncavo, formada por uma sequência de rochas sedimentares mesozóicas (cretáceas), terciárias e quaternárias pertencentes á bacia de afundamento tectônico. As rochas cristalinas fazem parte de um conjunto altamente metamorfizado, composto de gnaisses e granulitos, exibindo um solo residual avermelhado rico em óxido de ferro.

Um estudo hidrogeológico da área utilizando método geofísico de eletrorresistividade para caracterização da área é uma fonte de conhecimento para descrever e caracterizar a geologia local. Sendo assim, foram realizados dois caminhamentos elétricos perpendiculares à linha da praia e um paralelo com o objetivo de inferir qualitativamente a topografia do embasamento cristalino, identificar o nível estático no aquífero e compartimentar feições geológicas, rochas básicas e ácidas.

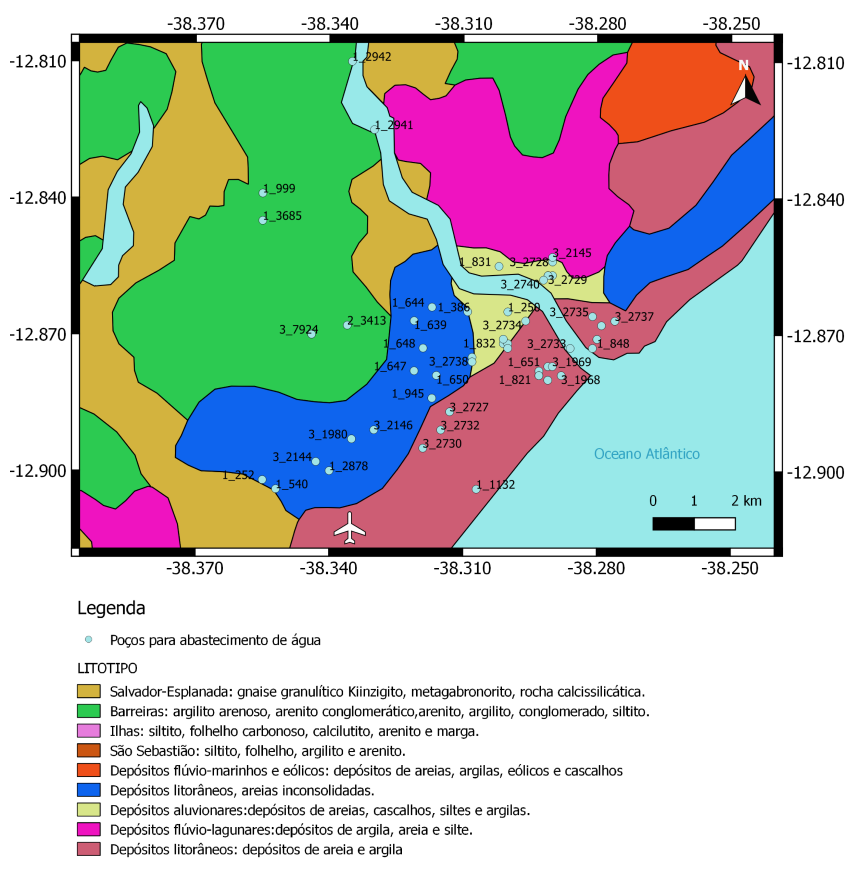

Figure 1: Litotipos e poços localizados na porção costal do município de Lauro de Freitas. (CPRM, 2017).

\section{Método Eletrorresistivo}

O método eletrorresistivo é baseado na medida da diferença de potencial entre dois pontos devido a excitação sobre um substrato rochoso de campo elétrico artificial criado pela injeção no solo de uma corrente elétrica contínua ou de frequência muito baixa.

A densidade de corrente $\mathbf{J}$ e a intensidade do campo 
elétrico $\mathbf{E}$ estão relacionadas pela lei de Ohm

$$
\mathbf{J}=\sigma \mathbf{E}
$$

Sendo $\sigma$, a condutividade elétrica do meio.

\section{Resistividade Aparente}

A resistividade aparente pode ser definida como a resistividade elétrica de um meio homogêneo que, substituindo um meio heterogêneo, provocaria as mesmas reações elétricas observadas nas mesmas condições geométricas dos eletrodos. Podemos escrever que nos pontos $M$ e $N$, os potenciais elétricos são dados por:

$$
\begin{aligned}
V_{M} & =\frac{\rho I}{2 \pi}\left(\frac{1}{A M}-\frac{1}{B M}\right), \\
V_{N} & =\frac{\rho I}{2 \pi}\left(\frac{1}{A N}-\frac{1}{B N}\right),
\end{aligned}
$$

sendo $\rho$ a resistividade elétrica do semi-espaço homogêneo, I, a corrente elétrica, $A M, A N, B M$ e $B N$, as distâncias entre os pontos $A$ e $B$ para os pontos $M$ e $N$. Então a diferença de potencial $\Delta V$ pode ser é expressa por:

$$
\Delta V=V_{M}-V_{N}=\frac{\rho I}{2 \pi}\left(\frac{1}{A M}-\frac{1}{B M}-\frac{1}{A N}+\frac{1}{B N}\right) .
$$

A partir daí, podemos chegar ao valor da resistividade do semi-espaço, com conhecimento dos valores de $\Delta V$, I e as distâncias $A M, B M, A N$ e $B N$. Para isso, é necessário fazer o uso da expressão anterior invertida para $\rho$, ou seja,

$$
\rho=\frac{\Delta V}{I}\left\{\frac{2 \pi}{\frac{1}{A M}-\frac{1}{B M}-\frac{1}{A N}+\frac{1}{B N}}\right\}
$$

\section{Caminhamento Elétrico}

O método do caminhamento elétrico (CE), Figura 2, tem aplicabilidade em pesquisas que tem como objetivo determinar descontinuidades laterais na distribuição de resistividade elétrica em subsuperfície (Braga, 1999). Tais descontinuidades podem ser dadas por diques, sills, contatos geológicos, fraturas ou corpos mineralizados. $\mathrm{O}$ CE também é bastante utilizado em estudos ambientais para identificar plumas de contaminação e aquíferos.

\section{Arranjo de Eletrodos do Caminhamento: Dipolo-Dipolo}

O arranjo dipolo-dipolo, é usado tanto no caminhamento elétrico, quanto em sondagens elétricas verticais. Nesta configuração os eletrodos de injeção de corrente $A B$ e de potencial $M N$ são dispostos em uma linha e o arranjo é definido pelos espaçamentos entre os eletrodos. A profundidade de investigação cresce com o distanciamento entre os eletrodos de potencial e os de corrente e, teoricamente, corresponde a $R / 2$, , sendo $R$ a máxima distância entre os pontos centrais de $A B$ e $M N$. As medidas são efetuadas em várias profundidades de investigação $(n), n=1,2,3,4,5 \ldots$ e posicionadas no ponto de intersecção entre uma linha que parte do centro do arranjo de eletrodos $A B$ e outra que parte de centro do arranjo $M N$, com ângulos de $45^{\circ}$ (Figura 2).

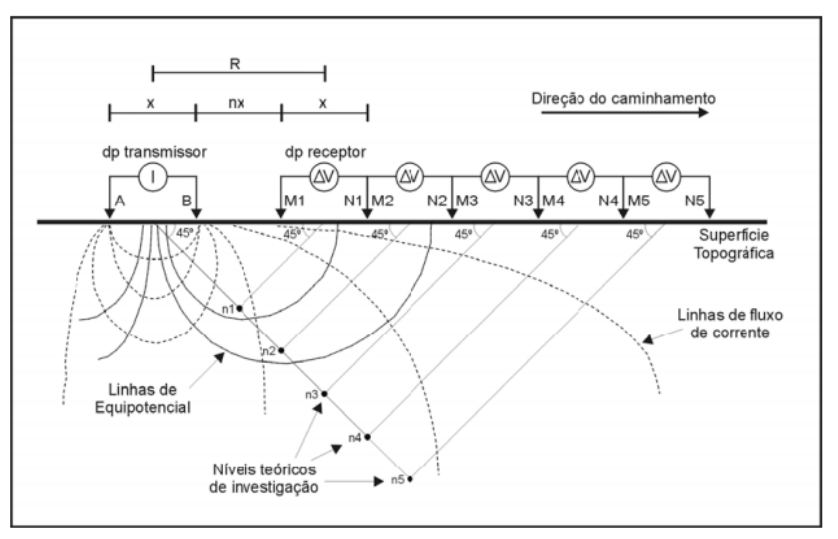

Figure 2: Técnica de caminhamento elétrico utilizando arranjo dipolo-dipolo (Braga, 1999).

\section{Aquisição dos dados}

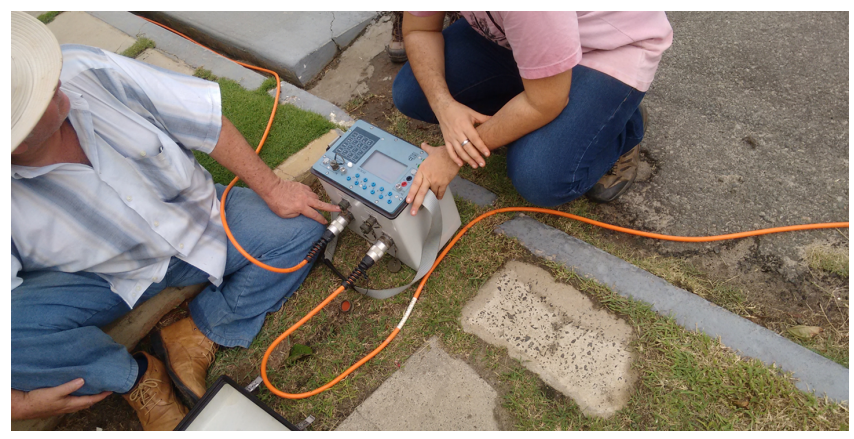

Figure 3: Imagem do momento da aquisição dos dados, mostra que a zona é urbanizada e pavimentada. As linhas foram posicionadas sobre o calçamento.

Os dados que constam neste trabalho foram adquiridos pela técnica de caminhamento elétrico, usando o arranjo dipolo-dipolo. O equipamento utilizado foi o resistivímetro modelo SYSCAL PRO, fabricado pela Iris Instruments, de propriedade do CPGG/UFBA. Este aparelho é composto por uma unidade transmissora e outra receptora, sendo que, elas tem configurações para realizarem leituras simultâneas da resistividade e cargabilidade aparentes, após inserir corrente elétrica, através de eletrodos inseridos no solo (Figura 3).

Primeiro foi realizado o caminhamento elétrico na rua Praia de Marambaia que foi chamado de linha 1, depois foi realizado o caminhamento elétrico na rua Praia Vermelha que foi chamada de linha 2 e por último foi realizado o caminhamento elétrico em parte da avenida Copacabana que é perpendicular as outras duas ruas citadas e paralela a linha da praia, sendo que, essa última foi chamada de linha 3. Os três caminhamento elétricos foram realizados durante o dia 29 de maio de 2017, sendo escolhido o arranjo dipolo-dipolo com espaçamento de 5 em 5 metros entre os eletrodos. A linha 1 teve 100 metros de extensão e as linhas 2 e 3 tiveram 120 metros de extensão.

Como dados complementares na interpretação, utilizamos duas SEV's (Figura 5 e Figura 6) de um trabalho, prévio feito na vizinhança, realizadas com arranjo Schlumberger 
e distâncias de afastamentos máximos entre os eletrodos de corrente elétrica e o centro da $S E V, A B / 2$ máximas, variando entre 100 e 500 metros. A intensa urbanização da área impossibilitou afastamentos maiores, produzindo a identificação de topo de camada até 20 e $24 \mathrm{~m}$ para as SEV12 e SEV17 respectivamente (como denominadas pelo autor do trabalho). As SEV's estão próximas da área de estudo (Figura 4) e fornecem uma base informativa sobre quantidade de camadas, espessura e resisitividade elétrica de cada horizonte geológico.

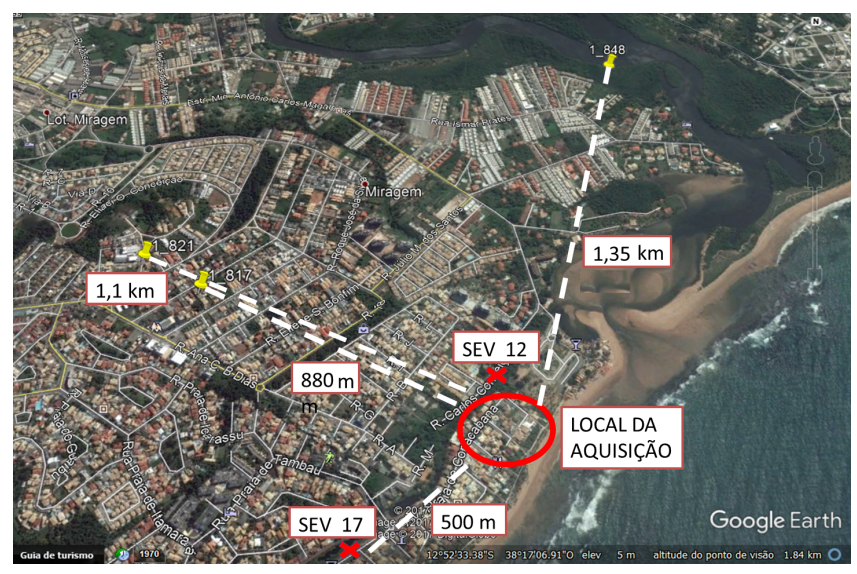

Figure 4: Localização da área estudada, mostrando a localização dos poços de água correlacionado e também as SEV'S(obtida no Google Earth e modificada)

Outra fonte de dados para embasar a interpretação, são um conjunto de 3 perfis estratigráficos (Figura 7), que mostram que o aquífero local é do tipo cristalino de topo entre 7-10m de profundidade. As fichas informativas dos poços, evidenciam uma cobertura arenosa sobre rocha cristalina alterada de topo em aproximadamente 7-9 m.

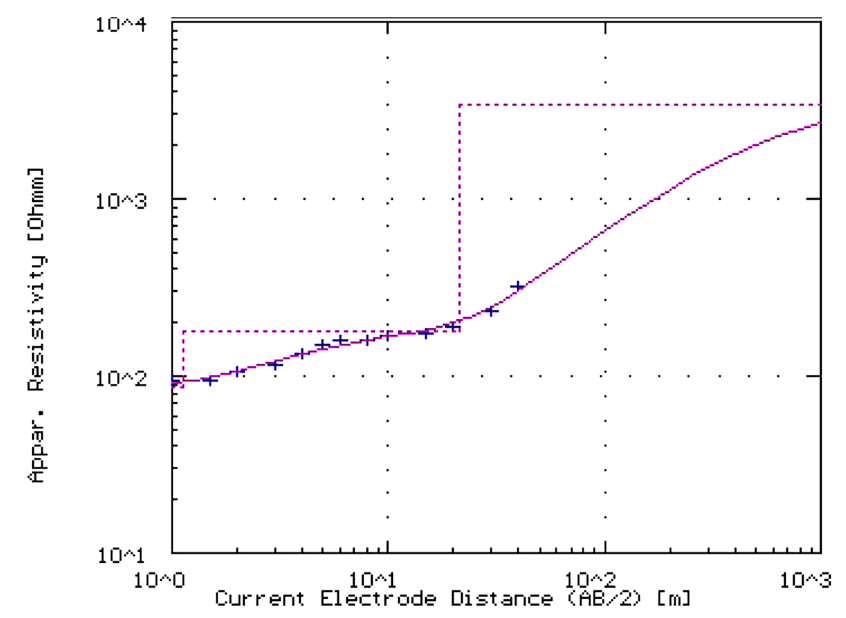

Figure 5: Perfil de resistividade da SEV 12: resistividade aparente medida (cruz azul) e invertida (linha contínua roxa).(Extraído de Santiago,2002)

\section{Processamento dos Dados}

Como parte do procedimento padrão, os dados da aquisição foram retirados do equipamento através

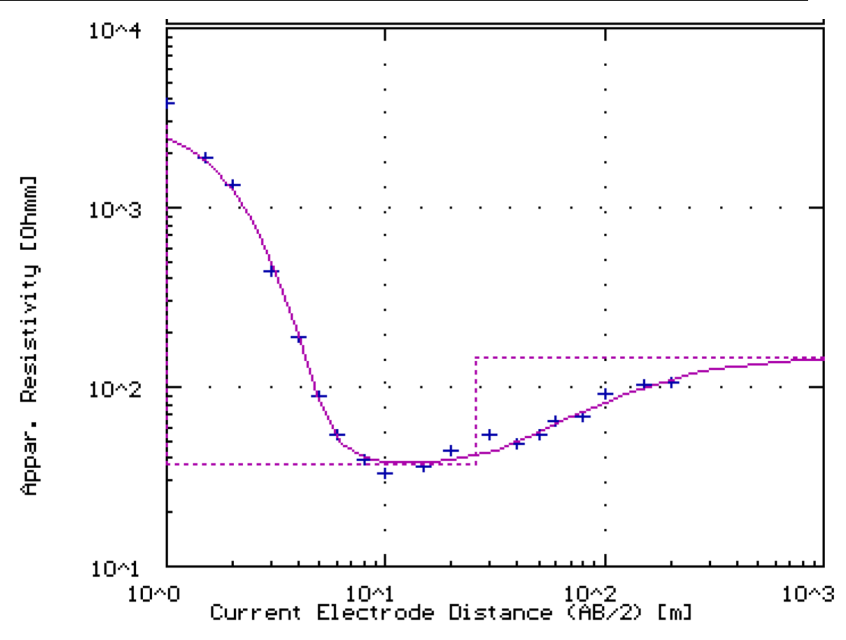

Figure 6: Perfil de resistividade da SEV 17: resistividade aparente medida (cruz azul) e invertida (linha contínua roxa)(Extraído de Santiago, 2002).

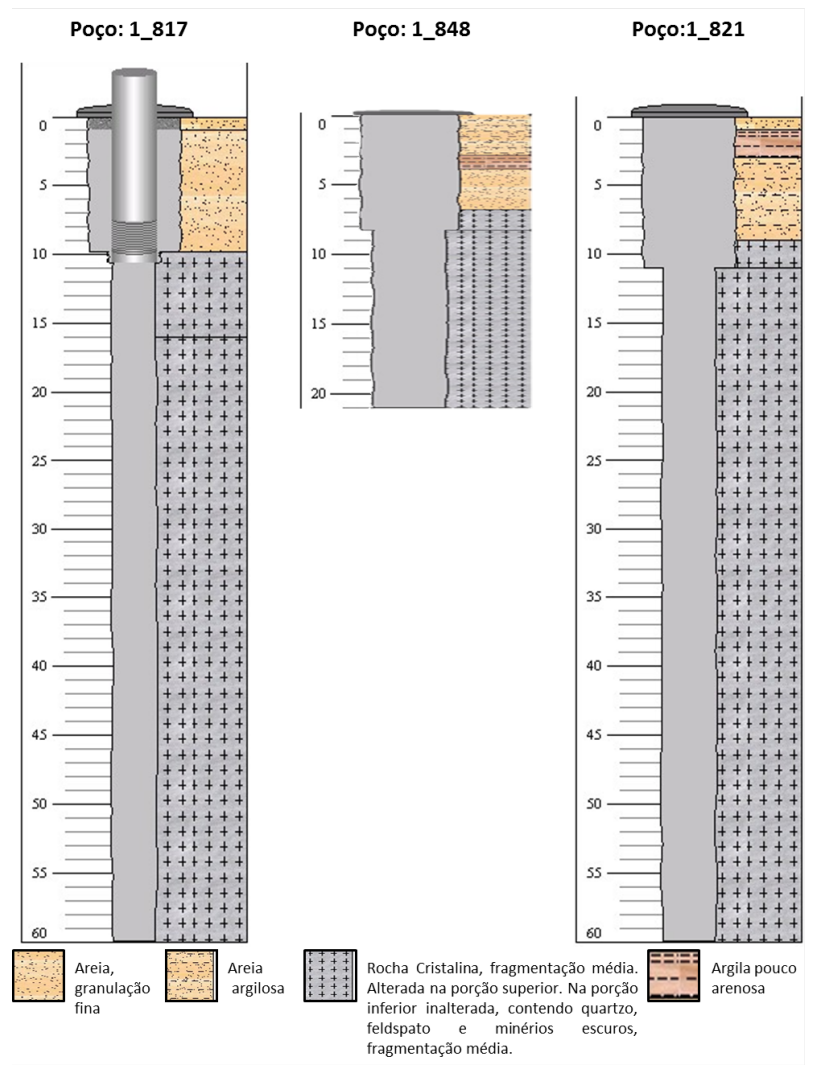

Figure 7: Perfis litologicos de poços vizinhos às linhas de levantamento (CERB, 2017).

do software Prosys 2, fornecido juntamente com o equipamento, e posterioemente organizados como .dat de acordo com a configuração de leitura dos programas de inversão RES2DINV e ELRIS2D no qual é feita a plotagem dos dados medidos e também a modelagem inversa. As medidas de resistividade geralmente são mostradas e interpoladas (contornos de isovalores) sob a forma de pseudoseções (Hallof, 1957). Como um dos 
objetivos do trabalho era testar e comparar os resultados de modelagem entre o RES2DINV, já muito utilizado na geofísica e o ELRIS2D, um programa escrito na linguagem matlab, uma adaptação ao formato de leitura do mesmo se fez necessária. Um pré-processamento necessário foi a remoção de medidas de resistividade aparente negativas e medidas que se repetiam, essas medidas poderiam gerar erros na inversão ou aumento desproporcional do erro de ajuste.

Para o processamento dos dados das SEV's, segundo (Santiago, 2002), houve também um pré-processamento, que consistiu da retirada de pontos anômalos isolados identificados por visualização, depois foram utilizados métodos que atualmente estão em desuso, como: método do ponto auxiliar, que através de curvas auxiliares précalculadas, obtém as espessuras das camadas e as resistividades do meio. Após obter o modelo inicial através do método do ponto auxiliar, o processamento melhorou, fornecendo maiores informações sobre as resistividades. $\mathrm{Na}$ modelagem foi usado o software RESIST, que executa a inversão automática dos perfis por um processo iterativo de otimização por mínimos quadrados que geram os parâmetros finais resultantes, otimizando o processamento, com resultados de melhor ajuste, possibilitante confiabilidade na interpretação dos dados adquiridos.

\section{Resultados}
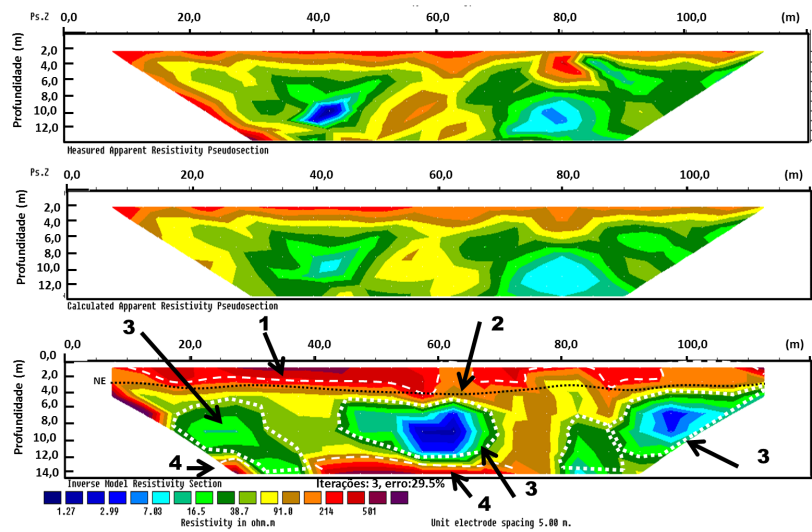

Figure 8: Linha 1, de cima para baixo: pseudoseção de resistividade, ajuste e seção invertida com linhas pontilhadas destacando volumes de rocha saturada.

Através dos dados adquiridos nos três caminhamentos elétricos realizados e informações das SEV'S 12 e 17, foi possível obter os modelos de inversão dos dados de resistividade aparente $1 \mathrm{D}$ e $2 \mathrm{D}$, mostrando as variações laterais e verticais da resistividade na subsuperfície. Sendo possível sugerir um modelo geológico para a área estudada da seguinte maneira.

i camadas superficiais não saturadas, delimitada na parte superior, indicada nas imagens com o número 1;

ii camadas arenosas com presença de argila, próximo ao nível estacionário, indicado nas figuras com número 2;
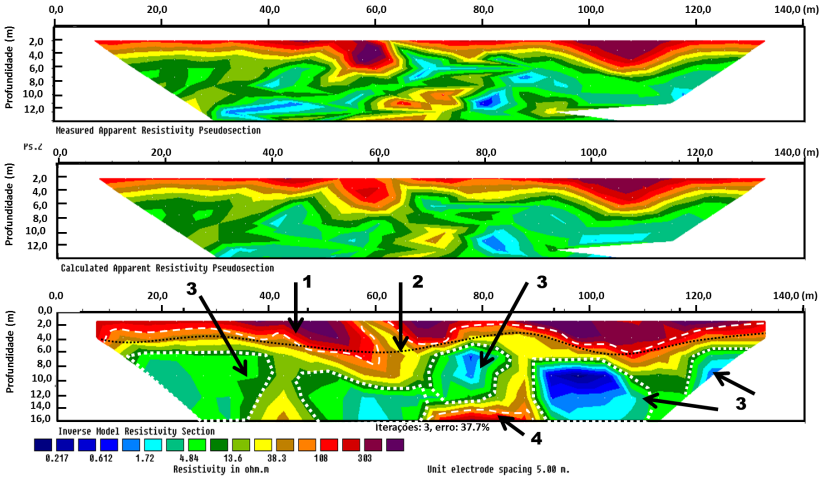

Figure 9: Linha 2, pseudoseção e ajuste seguidos da modelagem, onde estão em destaque 1- cobertura resistiva, 2- topo das rochas saturadas, 3- porções mais permeáveis e 4- embasamento.
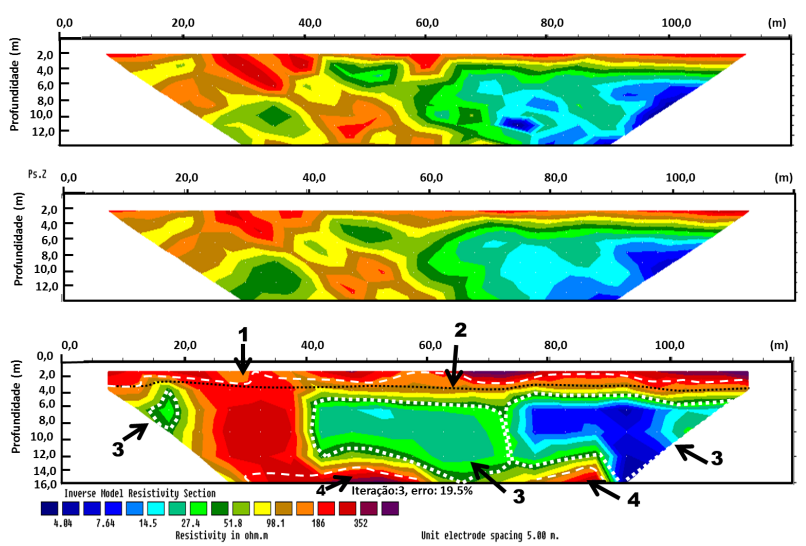

Figure 10: Linha 3, dados medidos e calculados, seguidos da seção de resitividade interpretada. Em destaque o nível dágua (pontilhado preto), e porções de rocha saturada (pontilhado branco).

iii Embasamento alterado e/ ou fraturado, indicado nas imagens com número 3, podendo ocorrer através de processos físicos ou químicos, como dissolução de minerais menos estáveis, como: feldspato, piroxênios e anfibólios;

iv Embasamento sem fraturas, indicado nas imagens com o número 4, sendo essa região impermeável.

Para o aquífero estudado, os resultados da modelagem da linha 01 (Figura 8), apresenta horizontes geológicos irregulares, as camadas superfíciais não saturadas correspondem a sedimentos arenosos não consolidados característicos de área litorânea (praias) que perdem água facilmente por evaporação, sendo registrado altos valores de resistividade elétrica. Logo a baixo desta, identificamos a zona saturada e nível estático do aquífero marcado por redução da resistividade elétrica em relação a camada sobrejacente. O embasamento alterado, que se extende aproximadamente da profundidade de 4 a 12 $\mathrm{m}$ de superfície irregular é destacado logo abaixo com menores valores de resistividade distribuídos de forma bandeada indicando zonas mais ácidas (numeração 3), 
por conseguinte mais resistentes a alterações quimicas e dissolução sendo quimicamente inerte. As intercalações com rochas mais instáveis indicam rochas menos silicosas, possivelmente composta de feldspatos e micas que são facilmente alterados sofrendo lixiviação de íons de sódio e potássio. A fragilidade desta porção enfatizada pela maior erosão na superfíe destas porções que nas vizinhanças silicosas, resultam em um acidentamento da superfície erosional com altos e baixos topográficos. Os parâmetro escolhidos para o levantamento, tais como arranjo e espaçamento, proporcionaram um mapemento de até $16 \mathrm{~m}$ de profundidade, tornando possível identificar apenas o topo do que seria oa rocha sã (indicado pelo numero 4). Perfis construtivos de poços da CERB (Figura 7) indicam que uma rocha cristalina estaria em profundidade aproximada de 16,8 , e $11 \mathrm{~m}$ de profundidade (perfis dispostos da esquerda para direita).

Considerando que as interpretações foram feitas também com auxílio de dados de poços de água do SIAGAS/ CPRM.

\section{Conclusões}

A utilização das técnicas de caminhamento elétrico, sondagem elétrica vertical e a correlação com dados de poços, apresentaram uma investigação satisfatória da área, possibilitando a criação de um modelo geológico pertinente. A análise dos resultados obtidos mostraram baixos valores de resistividades para a água subterrânea do local de estudo, o que permite concluir que esta apresenta alto teor de salinidade, o que a inviabiliza para consumo humano. Este resultado está de acordo com o que foi relatado pelos moradores dessa região que informaram que tal água apresenta alta salinidade.

As técnicas de eletrorresistividades utilizadas, caminhamento elétrico e sondagem elétrica vertical, são muito importantes para esse tipo de investigação pelo fato de possuir baixos custos comparados com outros métodos geofísicos. Outro ponto positivo é o fato de não necessitar de perfuração de poços para avaliar a qualidade da água, dispensando a investigação destrutiva, reduzindo assim, os custos para quem planeja a exploração hídrica de uma determinada área.

É recomendável que posteriormente seja utilizado o método magnético ou eletromagnético na área de investigação deste trabalho para que se tenha maior detalhamento geológico, e que se aumente o grau de confiança do modelo geológico sugerido.

\section{Referências}

Bortolozo, C. A. (2016) Inversão conjunta 1d e 2 d de dados de eletrorresistividade e TDEM aplicados em estudos de hidrogeologia na bacia do Paraná, Tese de Doutorado, Universidade de São Paulo.

Cabral, F. C. F. (1978) O uso dos isótopos do carbono no estudo das águas subterrâneas do calcário Bambuí - região central da Bahia, Dissertação de Mestrado, Universidade Federal da Bahia.

Gurgel, G. J. B. (2005) Modelagem numérica aplicada à análise da intrusão marinha na região sul da planície do Recife (PE), Dissertação de Mestrado, Universidade Federal de Pernambuco.
Hallof, P. G. (1957) On the interpretation of resistivity and induced polarization held measurements, Tese de Doutorado, Massachusetts Institute of Technology.

IBGE. (2017) Instituto Brasileiro de Geograa e Estatística. Disponível em: http://www .cidades.ibge.gov.br/xtras/perl.php?lang=codmun=291920 search=bahia-lauro-defreitas, acesso em: 15 de março de 2017.

Machado, R. (2008) Aplicação do método eletrorrestivo em ambiente aquático para o mapeamento do subfundo do Rio São Francisco, Dissertação de Mestrado, Universidade de São Paulo.

Mota, S. U. S. (2004) Caracterização Hidrogeológica do Setor Oriental do Pólo Industrial de Camaçari Utilizando Geofísica Elétrica, Dissertação de Mestrado, Universidade Federal da Bahia.

Santiago, Ricardo Cavalcanti (2002) Avaliação geofísica do sistema aquífero da área costerira entre Ipitanga e Rio Joanes, Trabalho de Graduação, Universidade Federal da Bahia. 